\title{
Green Synthesis of Silver Nanoparticles from Capitula extract of Some Launaea (Asteraceae) with Notes on their Taxonomic Significance
}

\author{
Momen M. Zareh(1), Nivien A. Nafady(2), Ahmed M. Faried (2)\# and Mona H. \\ Mohamed $^{(2)}$ \\ (1)Boilogy Dept., Faculty of Applied Science, Umm Al-Qura University, Makkah, Saudi \\ Arabia and ${ }^{(2)}$ Botany \& Microbiology Dept., Faculty of Science, Assiut University, \\ Assiut, Egypt.
}

\begin{abstract}
ATA are used to re-assess the relationships between certain species of the genus Launaea Cass. belonging to the family Asteraceae. Taxonomic diversity of 10 taxa belonging to 8 species and 2 subspecies of Launaea Cass. is provided using morphological criteria concerned with vegetative and reproductive organs in addition to FTIR spectroscopy. Ecofriendly silver nanoparticles were synthesized from Launaea's capitula extract and characterized by FTIR spectroscopy. NTSYS-pc software was used in order to analyze the data of FTIR spectroscopy and morphological characters. FTIR technique was used to recognize the functional groups of the active compound according to the peak value in Infrared radiation region. Cluster analysis based on FTIR data divided the ten studied taxa into three major groups; the first group comprises the four species of sect. Microrhynchus ( $L$. nudicaulis, L. intybacea and $L$. massauensis) and sect. Launaea (L. capitata), the second group comprises the four subspecies of L. angustifolia and L. fragilis; the third group comprises the two-allied species (L. mucronata and $L$. cassiniana). FTIR technique found to be a rapid and accurate method for differentiating between Launaea taxa under investigation.
\end{abstract}

Keywords: Launaea, Asteraceae, FTIR, Spectroscopy, Systematic, Silver nanoparticles.

\section{Introduction}

Taxonomists always found a problem in the systematic of the genus Launaea due to its confusing taxonomic history (Zareh et al., 2016a). Launaea Cass. comprises 55 species and it is mainly distributed in the S. Mediterranean, Africa, and SW Asia (Kilian, 1997). Until that time, several species today included in Launaea had already been described but placed in such different genera as Chondrilla, Lactuca, Leontodon, Prenanthes and Sonchus. For the most of the $19^{\text {th }}$ century, the present day Launaea species were dispersed and moved chiefly between Sonchus and Lactuca. According to Zareh et al. (2016a), the genus Launaea was represented in the flora of Egypt by twelve species and being the largest genus of the tribe Cichorieae.

There is a worldwide interest in synthesis of silver nanoparticles from environmentally friendly materials such as bacteria, enzymes, fungi and leaf extract (Bhainsa \& D'souza, 2006; Jain et al., 2009; Saifuddin et al., 2009 and Willner et al., 2007). Green synthesis of silver nanoparticles
(AgNPs) provides numerous benefits due to its environmental friendly effect as well as its lowcost. Recently, several literatures pay attention to the importance of using plant extract to synthesis silver nanoparticles (Dhand et al., 2016; Francis et al., 2017; Gomathi et al., 2017; Kumar et al., 2014; Saravanakumar et al., 2017 and Singh et al., 2010).

Mondal et al. (2011) synthesized ecofriendly silver nanoparticles from $\mathrm{AgNo}_{3}$ by the latex of six different plant taxa belonging to six different families; they proposed to use this modern technique for the taxonomy of angiosperms based on the ability of plants to synthesis silver nanoparticles which are variable in concentration, shape and size.

Chemotaxonomy has been strongly inclined the whole field of biology, which is also very useful for plant taxonomy (Mariswamy et al., 2012). FTIR spectroscopy (Fourier transform infrared) is a non-invasive, fast and a high-resolution analytical technique for recognized different types of chemical bonds by producing the infrared absorption spectrum that is like a molecular fingerprint (Griffiths \& de Haseth, 1986 and Smith,

\#Corresponding author email: faried55@yahoo.com

DOI: $10.21608 /$ ejbo.2018.1375.1111

C2018 National Information and Documentation Centre (NIDOC) 
1996). The application of a combination with numerical methodologies, FTIR is recommended and has many advantages. This technique has been successfully used for classifying of aged and normal soybean seeds (Kusama et al., 1997 and Wang et al., 2002). One of the most important applications of the IR spectroscopic study is the diagnostic value in establishing the occurrence of certain organic constituents in the plants (Velmurugan, 2006).

Recently, FTIR has been introduced as a metabolic fingerprinting tool for the plant sciences (Parveen et al., 2007 and Shen et al., 2008). In such an attempt, either the variation of the intensity or the frequency shift of some characteristic absorption bands can be used. According to Kim et al. (2004), the FTIR approach is strongly recommended to use in chemotaxonomic classification of flowering plants. The coming decades are likely to bring new approaches which will change our understanding of plants architecture and structure what is currently known in model organisms (Heywood et al., 2007).

In this study, ecofriendly silver nanoparticles were synthesized from capitula extract of eight closely similar taxa of Launaea (Table 1) and illustrate the relationships among the study taxa based on its chemical characteristics using FTIR approach in conjunction with the morphological characters.

\section{Materials and Methods}

Morphological studies were carried out on fresh material and voucher specimens deposited in different Egyptian herbaria: CAI, CAIM and ASTU. The investigated specimens used in morphological and FTIR spectroscopic studies are summarized in Table 1.

For observation the silver nanoparticles, the method followed Eri et al. (2014) with little modification as follows: The extract was made using $1 \mathrm{~g}$ of capitula; the capitula were cleaned thoroughly using distilled $\mathrm{H}_{2} \mathrm{O}$ and cut into small pieces, the capitula sample then added to $100 \mathrm{ml}$ of dist. $\mathrm{H}_{2} \mathrm{O}$ and then boil for $5 \mathrm{~min}$. The solution was then left to cool to normal temperature (approximately $25^{\circ} \mathrm{C}$ ). Following this step, the extract was then filtered through the filter paper.

The silver nitrate $\left(\mathrm{AgNO}_{3}\right)$ used in this study was obtained from sigma Aldrich, $40 \mathrm{ml}$ of capitula extract was added to $60 \mathrm{ml}$ of $10^{-3} \mu \mathrm{AgNO}_{3}$ solution and the reaction was left to take place at room temperature. The formation of silver nanoparticles was indicated by the changing the coloure from colouress to yellow and finally to dark brown. The solution then centrifuges to collect the silver nanoparticles. The nanoparticles were washed two times using distilled $\mathrm{H}_{2} \mathrm{O}$.

The formation of silver nanoparticles by capitula extract were scanned in the $300-900 \mathrm{~nm}$ wavelength rang using a double beam spectrophotometer (Perkin-Elmer lambda 750 spectrophotometer). The FTIR spectrum was obtained in the mid IR region of $400-4000 \mathrm{~cm}^{-1}$. The dried experimental sample was directly placed on the potassium bromide crystals and the spectrum was recorded in transmittance mode.

\section{Data scoring and analysis}

The analyses were carried out using NTSYSpc2 software. A cluster analysis was performed using the average taxonomic distance and UPGMA clustering. The correlation coefficient between the tree matrix and the distance matrix was calculated in order to examine how the cluster analysis fits the distance matrix.

For FTIR, each separate character was scored as present (1) or absent (0) for calculating different coefficients such as Simple Matching (SM), Sorensen-Dice (SD) and Jaccard (J). All detectable and clear characters were scored. Two phenograms were constructed, the first was based on morphological criteria and the second was based on combined characters of FTIR (Fig. 1 and 2).

\section{$\underline{\text { Results }}$}

The studied 76 morphological characters concerned with stem, leaves, stipules, inflorescence, flowers, capsules, seeds and embryo were studied, and the morphological criteria used in computer analysis were indicated in (Table 2 and Appendix 1). The cluster analysis based on morphological data (Fig. 1) divided the studied taxa into two major groups; the first group (A) comprises the species of sect. Zollikoferia which characterized by papillose marginal achenes and absence of pappus disk (namely: L. angustifolia, L. fragilis L. mucronata and $L$. cassiniana); the second group comprises the species of the other two sections (Launaea and Microrhynchus) that characterized by wrinkled or weakly papillose marginal achenes (Zareh et al., 2016b). 
TABLE 1. Plants used in FTIR analysis.

\begin{tabular}{|c|c|c|}
\hline NO. & Taxa & Locality \\
\hline 1 & Launaea nudicaulis (L.) Hook. f. & $\begin{array}{l}\text { Suez, Wadi Hagol, 13.4.2010, Zareh \& Aboul-Ela s.n. } \\
\text { (ASTU) }\end{array}$ \\
\hline 2 & Launaea intybacea (Jacq.) Beauverd & $\begin{array}{l}\text { Gebel Elba, Wadi Laseitit, 7.2.1962, Täckholm et al. } \\
1687 \text { (CAI) }\end{array}$ \\
\hline 3 & Launaea massauensis (Fresen.) Sch.Bip. ex Kuntze & $\begin{array}{l}\text { Marsa Allam, Wadi Abu Ghusun, 7.3.1989, Zareh \& } \\
\text { Fargali s.n. (ASTU) }\end{array}$ \\
\hline 4 & Launaea capitate (Spreng.) Dandy & $\begin{array}{l}\text { Marsa Matrouh, AL-Omyed, 5.4.2012, Zareh \& Aboul- } \\
\text { Ela s.n. (ASTU) }\end{array}$ \\
\hline 5 & $\begin{array}{l}\text { Launaea angustifolia (Desf.) Kuntze subsp. } \\
\text { angustifolia }\end{array}$ & $\begin{array}{l}\text { Alamein - Alexandria road, 10.4.2010, Zareh \& Aboul- } \\
\text { Ela s.n. (ASTU) }\end{array}$ \\
\hline 6 & $\begin{array}{l}\text { Launaea angustifolia (Desf.) Kuntze subsp. arabica } \\
\text { (Boiss.) N. Kilian }\end{array}$ & The Siwa Oasis, 29.12.1969, Zahran s.n. (CAI) \\
\hline 7 & Launaea fragilis (Asso) Pau subsp. fragilis & $\begin{array}{l}\text { Al Alamein, Sidi Abdel Rahman, 4.4.2012, Zareh \& } \\
\text { Aboul-Ela s.n. (ASTU) }\end{array}$ \\
\hline 8 & $\begin{array}{l}\text { Launaea fragilis (Asso) Pau subsp. tenuiloba } \\
\text { (Boiss.) Zareh \& Mohamed }\end{array}$ & $\begin{array}{l}\text { Alexandria-Matrouh, Kilo137, 4.4.2012, Zareh \& } \\
\text { Aboul-Ela s.n. (ASTU) }\end{array}$ \\
\hline 9 & Launaea mucronata (Forssk.) Muschl. & $\begin{array}{l}\text { Suez, Wadi Hagol, 13.4.2010, Zareh \& Aboul-Ela s.n. } \\
\text { (ASTU) }\end{array}$ \\
\hline 10 & Launaea cassiniana (Jaub. \& Spach) Kuntze & $\begin{array}{l}\text { Mersa Alam, Wadi Abu Ghusun, 8.3.1989, Zareh \& } \\
\text { Faraghali s.n. (ASTU) }\end{array}$ \\
\hline
\end{tabular}

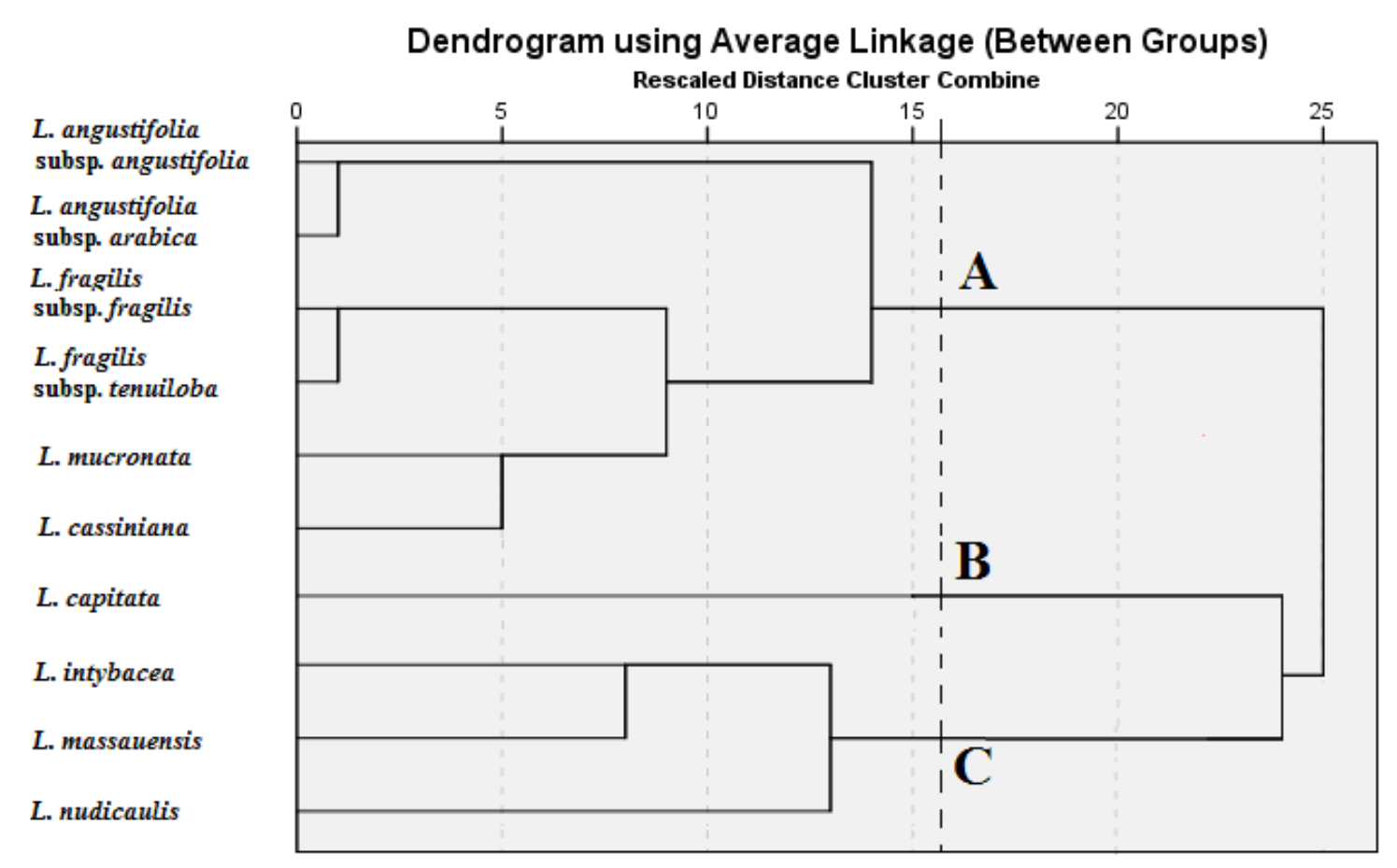

Fig. 1. Dendrogram illustrating the relationships among the 10 studied taxa of Launaea based on the morphological characters. A, sect. Zollikoferia; B, sect. Launaea and C, sect. Microrhynchus. 


\section{Dendrogram using j̉verage Linkage (Between Groups) \\ Rescaled Distance Cluster Combine}

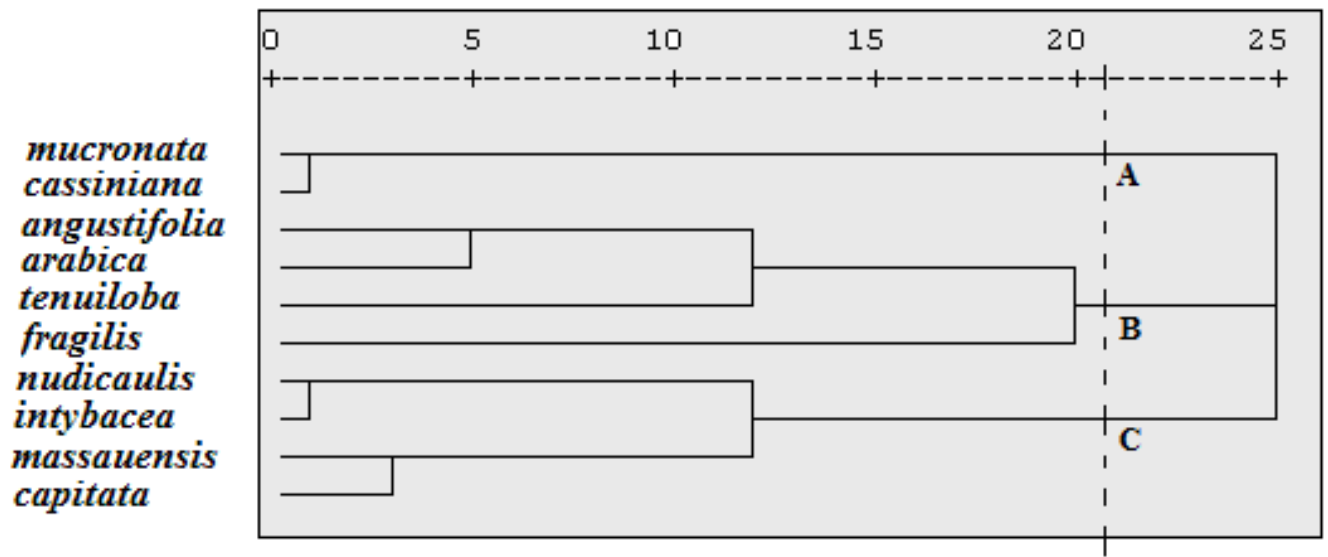

Fig. 2. Dendrogram illustrating the relationships among the 10 studied taxa of Launaea based on the FTIR characters.

TABLE 2. Characters and character state used in morphological analysis

\begin{tabular}{|c|c|c|c|c|c|}
\hline & Character \& character state & & $\begin{array}{c}\text { Character \& character } \\
\text { state }\end{array}$ & & Character \& character state \\
\hline \multirow[t]{4}{*}{1} & Habit & 14 & Basal leaves apex & 27 & Head long at anthesis \\
\hline & 1. Annual & & 1. Acute & & 1. $8-9 \mathrm{~mm}$ \\
\hline & 2. Perennial & & 2. Obtuse & & 2. $10-12 \mathrm{~mm}$ \\
\hline & 3. Shrub & & 3. Acuminate & & 3. $15-20 \mathrm{~mm}$ \\
\hline \multirow[t]{3}{*}{2} & Plant nature & 15 & Cauline leaves blade & 28 & Head long at fruiting \\
\hline & 1. Spinescent & & 1. Reduced & & 1. $9.0-10.0 \mathrm{~mm}$ \\
\hline & 2. Spineless & & 2. Ovate & & 2. $10.5-12.0 \mathrm{~mm}$ \\
\hline \multirow[t]{5}{*}{3} & Growth pattern & & 3. Auriculate & & 3. $12.5-14.0 \mathrm{~mm}$ \\
\hline & 1. Prostrate & 16 & Cauline leaves apex & & 4. $15.0-20.0 \mathrm{~mm}$ \\
\hline & 2. Procumbent & & 1. Reduced & 29 & Involucral bracts margin \\
\hline & 3. Twining & & 2. Acute & & 1. Not scarious \\
\hline & 4. Erect & & 3. Obtuse & & 2. narrow scarious \\
\hline \multirow[t]{3}{*}{4} & Plant form & 17 & Early leaves margin & & 3. broadly scarious \\
\hline & 1. Scape-like & & 1. Sub-entire & 30 & Outer bracts apex nature \\
\hline & 2. Caulescent & & 2. Serrate-dentate & & 1. Fleshy \\
\hline \multirow[t]{3}{*}{5} & Plant base & 18 & Longer leaf length & & 2. Callous-tipped \\
\hline & 1. Woody & & 1. $(7-8 \mathrm{~cm})$ & & 3. Cartilaginous \\
\hline & 2. Herbaceous & & 2. $(9-10 \mathrm{~cm})$ & 31 & Outer bracts apex shape \\
\hline \multirow[t]{6}{*}{6} & Plant height & & 3. $(11-12 \mathrm{~cm})$ & & 1. Acute \\
\hline & 1. $( \pm 10 \mathrm{~cm})$ & & 4. $(15-16 \mathrm{~cm})$ & & 2. Obtuse \\
\hline & 2. $(( \pm 25 \mathrm{~cm})$ & 19 & Synflorescence & & 3. Acuminate \\
\hline & 3. $(( \pm 30 \mathrm{~cm})$ & & 1. Heads single & 32 & Outer bracts broad \\
\hline & 4. $(( \pm 40 \mathrm{~cm})$ & & 2. Heads glomerate & & 1. $1.5-2 \mathrm{~mm}$ \\
\hline & 5. $(( \pm 100 \mathrm{~cm})$ & 20 & Head broad & & 2. $\pm 3 \mathrm{~mm}$ \\
\hline \multirow[t]{3}{*}{7} & Branching & & 1. less than $2.5 \mathrm{~mm}$ & 33 & Outer bracts length \\
\hline & 1. From base & & 2. more than $4.0 \mathrm{~mm}$ & & 1. $1.5-2.5 \mathrm{~mm}$ \\
\hline & 2. Above & 21 & Peduncles long & & 2. $\pm 3 \mathrm{~mm}$ \\
\hline \multirow[t]{3}{*}{8} & Branch leafness & & 1. Absent & 34 & Median bracts shape \\
\hline & 1. Leafy & & 2. $0.3-0.8 \mathrm{~mm}$ & & 1. oblong-lanceolate \\
\hline & 2. Leafless & & 3. $1.0-2.5 \mathrm{~mm}$ & & 2. ovate-lanceolate \\
\hline \multirow[t]{5}{*}{9} & Branch texture & & 4. $30-70 \mathrm{~mm}$ & 35 & Median bracts apex nature \\
\hline & 1. Glabrous & 22 & $\begin{array}{l}\text { Receptacle diameter at } \\
\text { fruiting }\end{array}$ & & 1. Fleshy \\
\hline & 2. Tomentose & & 1. $1.5-2.0 \mathrm{~mm}$ & & 2. Cartilaginous \\
\hline & 3. Puberulent & & 2. $2.5-3.0 \mathrm{~mm}$ & 36 & Median bracts apex shape \\
\hline & 4. Glaucescent & & 3. $3.5-5.5 \mathrm{~mm}$ & & 1. acute \\
\hline \multirow[t]{2}{*}{10} & Leaf margin nature & & 4. $6.0-10 \mathrm{~mm}$ & & 2. obtuse \\
\hline & 1. White cartilaginous & 23 & Head shape & & 3. acuminate \\
\hline
\end{tabular}


TABLE 2 Cont.

\begin{tabular}{|c|c|c|c|c|c|}
\hline & Character \& character state & & $\begin{array}{c}\text { Character \& character } \\
\text { state }\end{array}$ & & Character \& character state \\
\hline & 2. Green fleshy & & 1. Cylindrical & 37 & Median bracts shape \\
\hline \multirow[t]{5}{*}{11} & Basal leaves presence & & 2. Broadly cylindrical & & 1. oblong-lanceolate \\
\hline & 1. Rosetted & & 3. Narrowly cylindrical & & 2. ovate-lanceolate \\
\hline & 2. Early deciduous & & 4. Campanulate & 38 & Median bracts broad \\
\hline & Basal leaves shape & 24 & Head color & & 1. $(1.5-2.0 \mathrm{~mm})$ \\
\hline & 1. Spathulate & & 1. Green & & 2. $(2.5-3.0 \mathrm{~mm})$ \\
\hline \multirow[t]{4}{*}{12} & 2. linear to linear-spathulate & & 2. Grayish green & & 3. $(3.5-4.0 \mathrm{~mm})$ \\
\hline & 3. Oblong & 25 & Head broad at anthesis & 39 & Median bracts length \\
\hline & 4. Lanceolate & & 1. $2-2.5 \mathrm{~mm}$ & & 1. $(4.0-5.0 \mathrm{~mm})$ \\
\hline & 5. Oblanceolate-elliptic & & 2. $4-5 \mathrm{~mm}$ & & 2. $(5.5-6.0 \mathrm{~mm})$ \\
\hline \multirow[t]{5}{*}{13} & Basal leaves margin & & 3. $6-7 \mathrm{~mm}$ & & 3. $(6.5-7.0 \mathrm{~mm})$ \\
\hline & 1. Entire & 26 & Head broad at fruiting & 40 & Inner bracts apex nature \\
\hline & 2. Dentate & & 1. $2-3 \mathrm{~mm}$ & & 1. Fleshy \\
\hline & 3. Sinuate-dentate to pinnatifid & & 2. $4-5 \mathrm{~mm}$ & & 2. Cartilaginous \\
\hline & $\begin{array}{l}\text { 4. Sinuate-dentate to } \\
\text { pinnatisect }\end{array}$ & & 3. $6-7 \mathrm{~mm}$ & & \\
\hline \multirow[t]{3}{*}{41} & Inner bracts apex shape & 52 & Achenes wings & 64 & Inner achenes color \\
\hline & 1. Acute & & 1. Not winged & & 1. Whitish \\
\hline & 2. Obtuse & & 2. 1-winged & & 2. Brown \\
\hline \multirow[t]{3}{*}{42} & Inner bracts broad & & 3. 2-3-winged & & 3. Yellow \\
\hline & 1. $1-1.5 \mathrm{~mm}$ & 53 & Achenes length & 65 & Inner achenes shape \\
\hline & 2. $2-2.5 \mathrm{~mm}$ & & 1. $2.0-3.0 \mathrm{~mm}$ & & 1. columnar \\
\hline \multirow[t]{5}{*}{43} & Inner bracts length & & 2. $3.5-4.0 \mathrm{~mm}$ & & 2. compressed \\
\hline & 1. $8.0-9.0 \mathrm{~mm}$ & & 3. $4.5-6.0 \mathrm{~mm}$ & 66 & Inner achenes apex \\
\hline & 2. $9.5-10.0 \mathrm{~mm}$ & & 4. $\quad 6.5-8.0 \mathrm{~mm}$ & & 1. cuspidate \\
\hline & 3. $10.5-12.0 \mathrm{~mm}$ & 54 & Achenes width & & 2. not cuspidate \\
\hline & 4. $12.5-15.0 \mathrm{~mm}$ & & 1. $\quad 0.5-0.6 \mathrm{~mm}$ & 67 & Inner achenes sculpture \\
\hline \multirow[t]{6}{*}{44} & Flowers per capitulm & & 2. $\quad 0.7-0.9 \mathrm{~mm}$ & & 1. smooth \\
\hline & 1. $6-10$ & & 3. $1-1.3 \mathrm{~mm}$ & & 2. wrinkled \\
\hline & 2. $15-20$ & 55 & Achenes base & & 3. papillose \\
\hline & 3. $25-33$ & & 1. obtuse teethed & 68 & Inner achenes ribs \\
\hline & 4. $35-50$ & & 2. not teethed & & 1. 4 main \\
\hline & 5. $\geq 55$ & 56 & Marginal achenes color & & 2. 5 main \\
\hline \multirow[t]{5}{*}{45} & Ligules length & & 1. whitich & & 1. 2 , distinct \\
\hline & 1. $\quad 4.0-6.0 \mathrm{~mm}$ & & 2. brown & & 2. not differentiated \\
\hline & 2. $\quad 6.5-8.0 \mathrm{~mm}$ & & 3. grey & 69 & Inner achenes secondary ribs \\
\hline & 3. $8.5-12.0 \mathrm{~mm}$ & & 4. blackish & & 1. 2, distinct \\
\hline & 4. $12.5-20.0 \mathrm{~mm}$ & & 5. yellow & & 2. not differentiated \\
\hline \multirow[t]{5}{*}{46} & Ligules width & 57 & Marginal achenes angles & 70 & Inner achenes ribs surface \\
\hline & 1. $0.8-1.5 \mathrm{~mm}$ & & 1. \pm 5 angular & & 1. transversally wrinkled \\
\hline & 2. $\quad 1.7-2.2 \mathrm{~mm}$ & & 2. \pm 4 angular & & 2. glabrous \\
\hline & 3. $2.4-2.8 \mathrm{~mm}$ & & 3. not angular & & 3. squamulose-papillose \\
\hline & 4. $3.0-5.5 \mathrm{~mm}$ & 58 & Marginal achenes length & 71 & Pappus long \\
\hline \multirow[t]{3}{*}{47} & Flowers tube length & & 1. shorter than inner & & 1. $5-6 \mathrm{~mm}$ \\
\hline & 1. $3.5-6.0 \mathrm{~mm}$ & & 2. longer than inner & & 2. $7-8 \mathrm{~mm}$ \\
\hline & 2. $\quad 6.5-9.0 \mathrm{~mm}$ & 59 & Marginal achenes shape & & 3. $9-10 \mathrm{~mm}$ \\
\hline \multirow[t]{4}{*}{48} & Anther length & & 1. columnar & 72 & Pappus \\
\hline & 1. $\quad 1.2-2.2 \mathrm{~mm}$ & & 2. compressed & & 1. persistent \\
\hline & 2. $2.5-3.2 \mathrm{~mm}$ & 60 & Marginal achenes apex & & 2. deciduous \\
\hline & 3. $\quad 3.5-5.5 \mathrm{~mm}$ & & 1. truncate & 73 & Pappus symmetry \\
\hline \multirow[t]{4}{*}{49} & Style-arms long & & 2. cuspidate & & 1. monomorphic \\
\hline & 1. $\quad 0.5-1 \mathrm{~mm}$ & 61 & $\begin{array}{l}\text { Marginal achenes } \\
\text { sculpture }\end{array}$ & & 2. dimorphic \\
\hline & 2. $3-3.5 \mathrm{~mm}$ & & 1. wrinkled & 74 & Pappus disk \\
\hline & 3. $3.5-4 \mathrm{~mm}$ & & 2. papillose & & 1. present \\
\hline \multirow[t]{3}{*}{50} & Style sweeping hairs color & 62 & Achenes secondary ribs & & 2. absent \\
\hline & 1. yellow & & 1. \pm 2 distinct & 75 & Inner setaceous \\
\hline & 2. blackish & & 2. not distinct & & 1. absent \\
\hline \multirow[t]{4}{*}{51} & Achenes symmetry & 63 & Achene ribs surface & & 2. small number \\
\hline & 1. sub homomorphic & & 1. hirsute at angles & & 3. large number \\
\hline & 2. heteromorphic & & 2. transversally wrinkled & 76 & Outer downy \\
\hline & & & 3. squamulose-papillose & & $\begin{array}{l}\text { 1. absent } \\
\text { 2. large number }\end{array}$ \\
\hline
\end{tabular}


The later group can be divided into two groups; one (B) comprises the species of sect. Launaea ( $L$. capitata) which are characterized by prostrate habit and aggregate heads; the second group (C) comprises the species of section Microrhynchus (L. nudicaulis, L. intybacea and L. massauensis) that can be separated based on pappus characters. This grouping agrees with regards of Kilian (1997) in which he arranged the studied species in three different tribes.

The cluster analysis based on FTIR data (Fig. 2) divided the ten studied taxa into three major groups; the first group comprises the four species of sect. Microrhynchus (L. nudicaulis, $L$. intybacea and L. massauensis) and sect. Launaea (L. capitata), the second group comprises the four subspecies of $L$. angustifolia and L. fragilis; the third group comprises the two allied species ( $L$. mucronata and L. cassiniana).

\section{Discussion}

Launaea cassiniana (Jaub. \& Spach) Kuntze was described by both Kilian (1997) and Boulos (2002) as L. mucronata subsp. cassiniana.
According to Täckholm (1974) and Zareh et al. (2016b), L. mucronata can easily distinguished from $L$. cassiniana by several morphological characters which summarized in Table 3.

On the other hand, L. tenuiloba (Boiss.) Kuntze is treated by Kilian (1997) and Boulos (2002) as a synonym to L. fragilis, the plant is differing in being procumbent, branched from base with subentire leaves and wrinkled inner achenes, thus it is treated here as L. fragilis subsp. tenuiloba (Boiss.) Zareh \& Mohamed comb. nov. (Zareh et al., 2016b).

According to Griffiths \& De Haseth (2007), FTIR approach allows distinguishing the whole range of infrared spectrum in the measurements of plant specimens. The biosynthesized silver nanoparticles were formed in several different shapes such as hexagonas, spherical, monodispersed and uniformly distributed. Capitulum release reducing agents into the solution which are responsible for the formation of silver nanoparticles. Also, the particles are not aggregate, which might be an indication to the presence of a capping agent.

TABLE 3. Morphological differences between $L$. mucronata and $L$. cassiniana

\begin{tabular}{lll}
\hline Launaea mucronata & Launaea cassiniana \\
\hline Involucre bracts & Marginal scarious & Non marginal scarious \\
Achenes length & Shorter than $6 \mathrm{~mm}$ & Longer than $6 \mathrm{~mm}$ \\
Achenes shape & Compressed, 5 -angeled & Columnar, not angular \\
Pappus & Hetero-morphic & Mono-morphic \\
\hline
\end{tabular}

TABLE 4. General band assignments of the average FTIR spectrum of plants

\begin{tabular}{ll}
\hline O-H stretching vibrations of hydroxyl groups & N-H stretching of aliphatic and aromatic amines \\
$\mathrm{H}-$-bonded alcohols, phenols & $\mathrm{C}=\mathrm{C}$ or C-N stretching of aromatic amines \\
$\mathrm{N}-\mathrm{H}$ stretching of $1^{\circ}$ and $2^{\circ}$ amines and amides & $\mathrm{C}-\mathrm{O}$ vibration \\
$\mathrm{C}-\mathrm{H}$ stretching of $\mathrm{CH} 2$ & $\mathrm{C}-\mathrm{Cl}$ bending \\
$(\mathrm{NH}) \mathrm{C}=\mathrm{O}$ stretching & $-\mathrm{C}-\mathrm{O}-\mathrm{C}$ \\
$\mathrm{NO}_{2}$ & $\mathrm{C}=\mathrm{C}$ stretching of alkene \\
$\mathrm{C}-\mathrm{N}$ stretching of aliphatic amines or to alcohols or phenols & $\mathrm{C}-\mathrm{N}$ stretching of aromatic and aliphatic amines \\
$\mathrm{O}-\mathrm{SI}-\mathrm{O}$ stretching & $\mathrm{C}-\mathrm{C}-\mathrm{N}$ asymmetric stretching vibration \\
$\mathrm{C}-\mathrm{H}$ stretching of aldehyde & $\mathrm{C}-\mathrm{N}, \mathrm{C}=\mathrm{N}$ stretching \\
$\mathrm{C}-\mathrm{N}$ stretching of primary and secondary amines & $\mathrm{C}-\mathrm{Cl}$ stretching \\
NH2 Wagging of amino & $\mathrm{C}-\mathrm{N}$ stretching of amino \\
Lead & $\mathrm{C}-\mathrm{H}$ stretching of alkane \\
\hline
\end{tabular}


The FTIR results adequate with the morphological characters; the species of the first group are characterized by wrinkled achenes and truncate base, the second are characterized by papillose achenes and horned base and the third groups are characterized by papillose achenes and tubular base. Nevertheless, the FTIR analysis showed that the two subspecies of $L$.
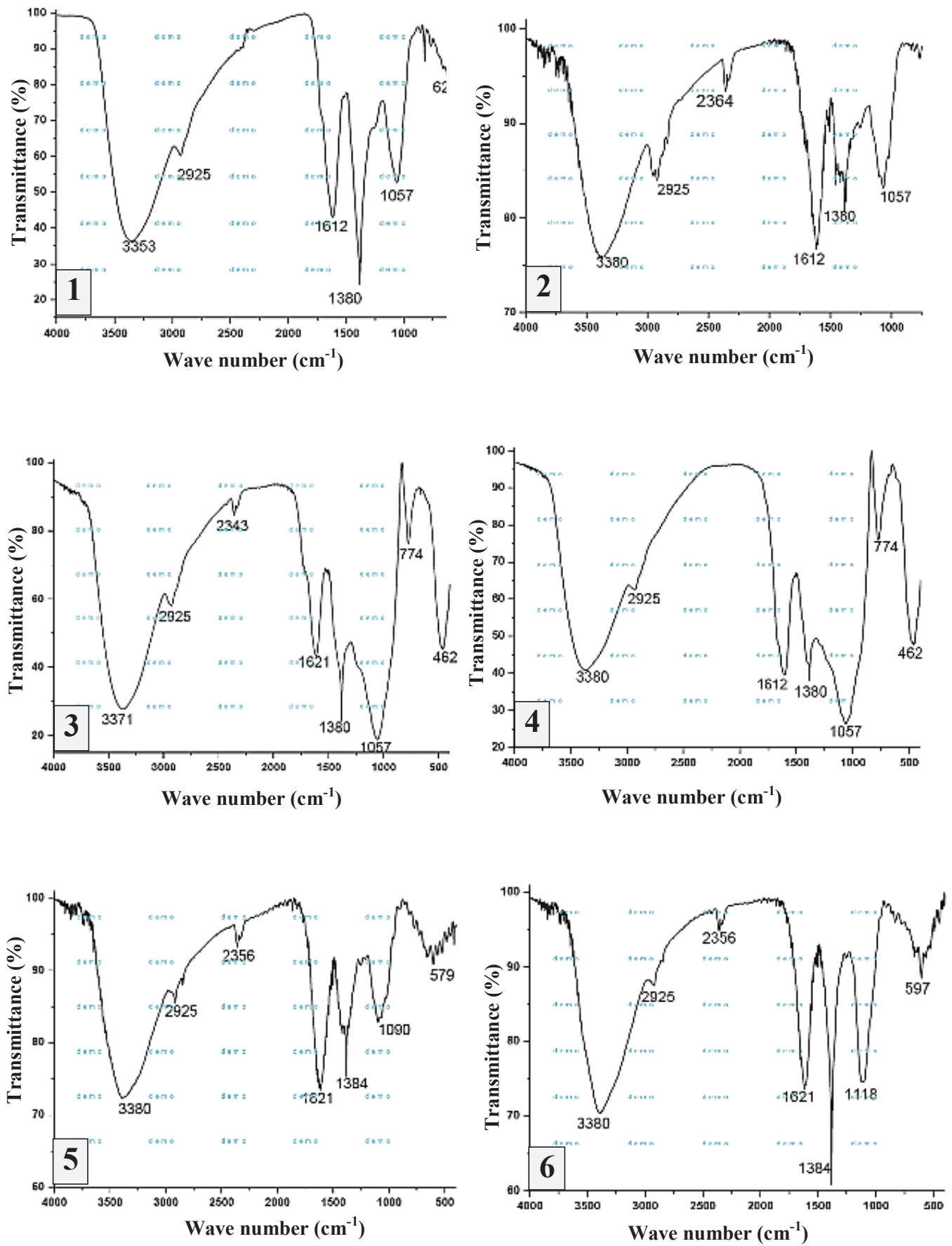

Fig. 3. FTIR spectra of the Launaea taxa. 1, L. nudicaulis; 2, L. intybacea; 3, L. massauensis; 4, L. capitate; 5, L. angustifolia subsp. angustifolia and $6, L$. angustifolia subsp. arabica.

Egypt. J. Bot. 58, No. 2 (2018)

fragilis (subsp. fragilis and subsp. tenuiloba) is apparently different in their components, this adequate with the treatment of Täckholm (1974) as two different taxa. The summary of the most general band assignments observed in the studied taxa are presented in Table 4 and Appendix 2, the FTIR spectra also are showed in Fig. 3 and 4.

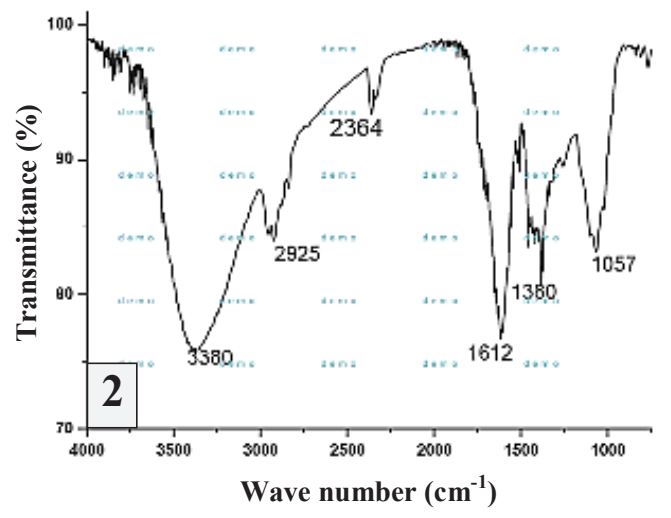

Wave number $\left(\mathrm{cm}^{-1}\right)$ 

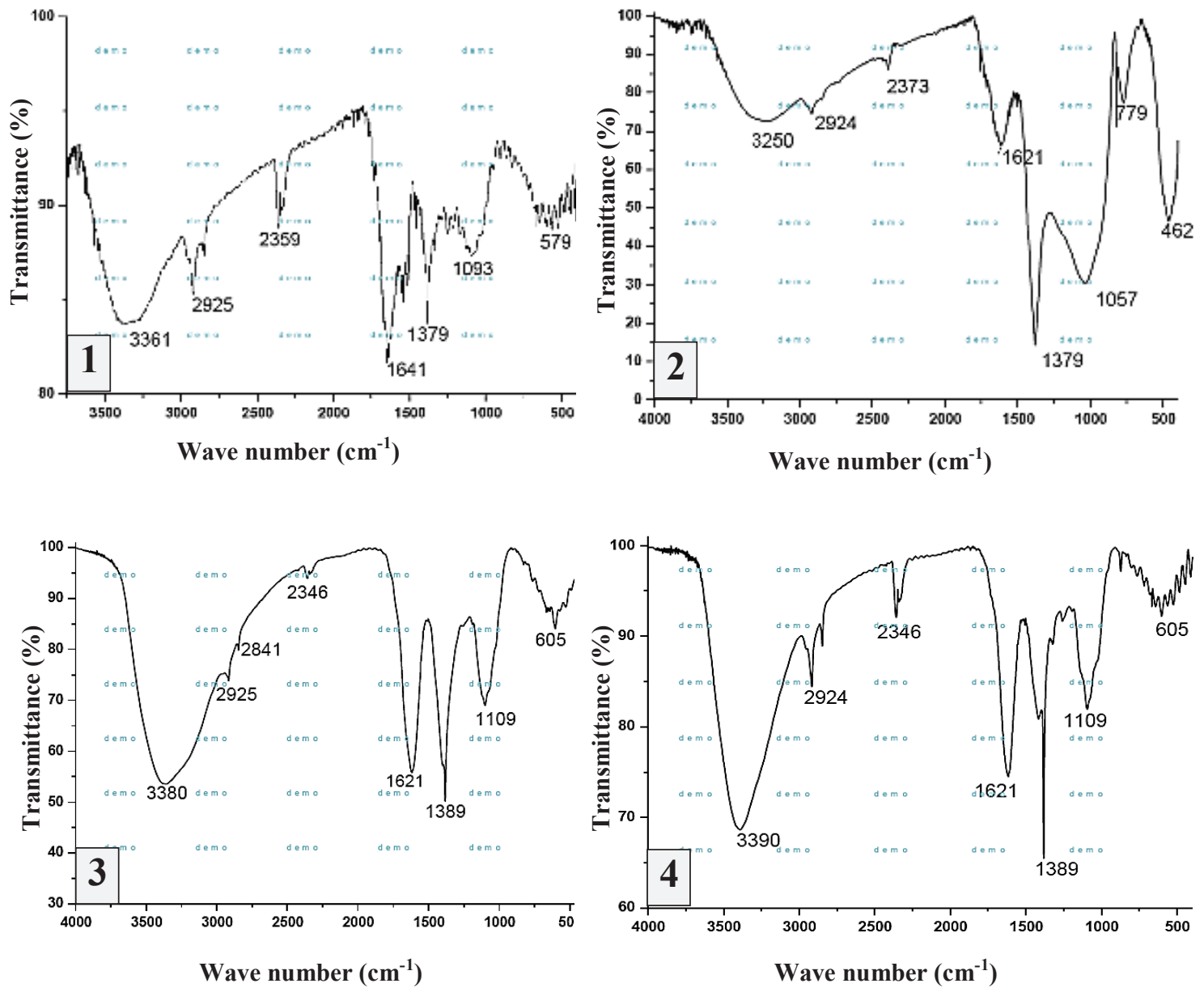

Fig. 4. FTIR spectra of the Launaea taxa. 1, L. fragilis subsp. fragilis; 2, L. fragilis subsp. tenuiloba; 3 , L. mucronate and 4, L. cassiniana.

\section{Conclusion}

Morphological and FTIR spectroscopic studies was applied on ten closely related taxa of the genus Launaea belonging to family Asteraceae. The functional groups of the active components were identified based on the peak value in Infrared radiation region for each taxon. The results show that the FTIR spectroscopic technique was found to be an accurate and a rapid method to differentiate between closely and confused related taxa and have provided a valid source of taxonomic evidence for addressing the relationships at the species taxonomic levels.

\section{References}

Bhainsa, K.C. and D'souza, S. (2006) Extracellular biosynthesis of silver nanoparticles using the fungus Aspergillus fumigatus. Colloids and Surfaces B: Biointerfaces, 47, 160-164.
Boulos, L. (2002) "Flora of Egypt (Verbenaceae Compositae)". Volum 3. Al Hadara Publishing, Egypt.

Dhand, V., Soumya, L., Bharadwaj, S., Chakra, S., Bhatt, D. and Sreedhar, B. (2016) Green synthesis of silver nanoparticles using Coffea arabica seed extract and its antibacterial activity. Materials Science and Engineering, C 58, 36-43.

Eri, G.K., Naik, M.C., Padma, Y., Ramana, M.V., Madhu, M. and Gopinath, C. (2014) Novel FT-IR spectroscopic method for the quantitation of atenolol in bulk and tablet formulations, Journal of Global Trends in Pharmaceutical Sciences, 5, 31750-31755.

Francis, S., Joseph, S., Koshy, E.P. and Mathew, B. (2017) Green synthesis and characterization of gold and silver nanoparticles using Mussaenda glabrata leaf extract and their environmental applications to dye degradation. Environmental Science and 
Pollution Research, 24(21), 17347-17357.

Gomathi, M., Rajkumar, P., Prakasam, A. and Ravichandran, K. (2017) Green synthesis of silver nanoparticles using Datura stramonium leaf extract and assessment of their antibacterial activity. Resource-Efficient Technologies, 3, 280-284.

Griffiths, P.R. and de Haseth, J.A. (1986) Fourier transform infrared spectroscopy. Science, 222, 297 302.

Griffiths, P.R. and De Haseth, J.A. (2007) "Fourier Transform Infrared Spectrometry". John Wiley \& Sons, Canada.

Heywood, V.H., Brummitt, R.K, Culham, A. and Seberg, O (2007) "Flowering Plant Families of the World". Firefly Books Ontario.

Jain, D., Daima, H.K., Kachhwaha, S. and Kothari, S. (2009) Synthesis of plant-mediated silver nanoparticles using papaya fruit extract and evaluation of their anti microbial activities. Digest Journal of Nanomaterials and Biostructures, 4, 557563.

Kilian, N. (1997) Revision of Launaea Cass. (Compositae, Lactuceae, Sonchinae). Englera, 17, 1-478.

Kim, S.C., Lu, C.T. and Lepschi, B.J. (2004) Phylogenetic positions of Actites megalocarpa and Sonchus hydrophilus (Sonchinae: Asteraceae) based on ITS and chloroplast non-coding DNA sequences. Australian Systematic Botany, 17, 73-81.

Kumar, D.A., Palanichamy, V. and Roopan, S.M. (2014) Green synthesis of silver nanoparticles using Alternanthera dentata leaf extract at room temperature and their antimicrobial activity. Spectrochimica Acta Part A: Molecular and Biomolecular Spectroscopy, 127, 168-171.

Kusama, T., Abe, H., Kawano, S. and Iwamoto, M. (1997) Classification of normal and aged soybean seeds by discriminant analysis using principal component scores of near infrared spectra. Journal of the Japanese Society for Food Science and Technology (Japan), 53, 313-319.

Mariswamy, Y., Gnanaraj, W.E. and Antonisamy, J.M (2012) FTIR spectroscopic studies on Aerva lanata (L.) Juss. ex Schult. Asian J. Pharm. Clin. Res. 5, 82-86.
Mondal, A.K., Mondal, S., Samanta, S. and Mallick, S. (2011) Synthesis of ecofriendly silver nanoparticle from plant latex used as an important taxonomic tool for phylogenetic interrelationship. Advances in Bioresearch, 2, 122-133.

Parveen,Z., Yulin, D., Saeed, M.K., Rongji, D., Ahamad, W. and Yu, Y.H. (2007) Anti-inflammatory and analgesic activities of Thesium chinense Turcz extracts and its major flavonoids, kaempferol and kaempferol-3-O-glucoside. Yakugaku Zasshi, 127, 1275-1279.

Saifuddin, N., Wong, C. and Yasumira, A. (2009) Rapid biosynthesis of silver nanoparticles using culture supernatant of bacteria with microwave irradiation. Journal of Chemistry, 6, 61-70.

Saravanakumar, A., Peng, M.M., Ganesh, M., Jayaprakash, J., Mohankumar, M. and Jang, H.T. (2017) Low-cost and eco-friendly green synthesis of silver nanoparticles using Prunus japonica (Rosaceae) leaf extract and their antibacterial, antioxidant properties. Artificial Cells, Nanomedicine, and Biotechnology, 45, 1165-1171.

Shen, J.B., Lue, H.F, Peng, Q.F., Zheng, J.F. and Tian, Y.M. (2008) FTIR spectra of Camellia sect. Oleifera, sect. Paracamellia, and sect. Camellia (Theaceae) with reference to their taxonomic significance. Journal of Systematics and Evolution, 46, 194-204.

Singh, A., Jain, D., Upadhyay, M., Khandelwal, N. and Verma, H. (2010) Green synthesis of silver nanoparticles using Argemone mexicana leaf extract and evaluation of their antimicrobial activities. Dig. J. Nanomater. Bios. 5, 483-489.

Smith, B. (1996) "Fourier Transform Infrared Spectroscopy". CRC Press, Boca Raton, Florida.

Täckholm, V. (1974) "Students' Flora of Egypt". Cairo University, Egypt.

Velmurugan., S. (2006) Spectroscopic studies on the status of redroot disease incidence and its variation in sugarcane (Saccharum officinarum) L., Ph.D. Thesis, Annamalai University, Annamalainagar India.

Wang, D., Ram, M. and Dowell, F. (2002) Classification of damaged soybean seeds using near-infrared spectroscopy. Transactions-American Society of 
Agricultural Engineers, 45, 1943-1950.

Willner, I., Basnar, B. and Willner, B. (2007) Nanoparticle-enzyme hybrid systems for nanobiotechnology. The FEBS Journal, 274, 302309.

Zareh, M., Faried, A. and Mohamed, M. (2016a) Revision of Launaea Cass. (Compositae) in Egypt with special references to cypselar diversity. Feddes Repertorium, 127, 1-16.
Zareh, M., Faried, A. and Mohamed, M.H. (2016b) Achene wall anatomy and surface sculpturing of Launaea Cass. (Compositae: Cichorieae) with notes on their systematic significance. Korean J. Pl. Taxon. 46, 187-198.

\section{التخليق الأمن لجسيمات الفضة الناتوجزيئية من مستخلص نورات بعض أنواع اللاونيا

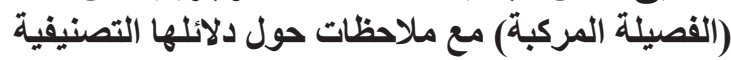

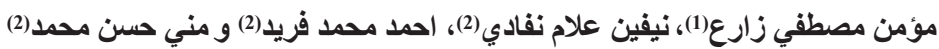

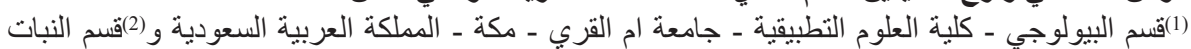

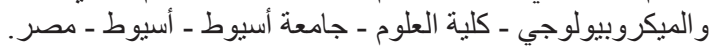

استخدمت النتائج في هذه الدراسة من اجل إعادة تقييم العلاقات بين بعض أنواع جنس اللاونيا التابع للفصيلة

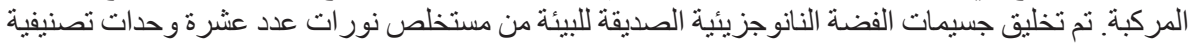

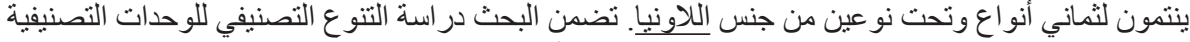

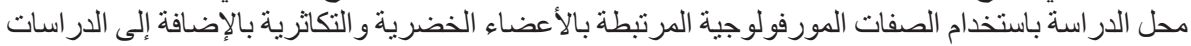
الطيفية باستخدام تقنية FTIR. تم استخدام برنامج NTSYS-pc لتقييم التحليل الإحصائي لجميع النتائج التي

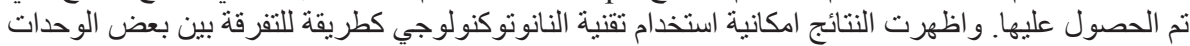

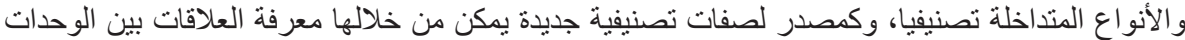
التصنيفية محل الدراسة. 